\title{
LAS NUEVAS TECNOLOGÍAS COMO HERRAMIENTA PEDAGÓGICA PARA FACILITAR LA COMUNICACIÓN
}

\author{
José Antonio del Barrio del Campo y María Rosa García Ruiz \\ Profesores y miembros del grupo de investigación “Isla de Mouro”. Universidad de \\ Cantabria.
}

\section{Resumen}

La formación a través de las Nuevas Tecnologías representa el presente y el futuro de cualquier sistema educativo. Teniendo en cuenta las posibilidades formativas que ofrecen, es necesario contar con nuevos ámbitos de formación para los docentes, de manera que puedan desarrollar su competencia profesional de forma equilibrada y con las mejores garantías de éxito. En este artículo se presenta una experiencia en la que tanto los actuales profesores como los futuros docentes tienen la posibilidad de completar su formación universitaria a través del “Aula de Oratoria de la Universidad de Cantabria”, convirtiéndose en grandes comunicadores.

\section{Palabras clave}

Oratoria- Enseñanza Virtual - Formación de docentes - Comunicación oral 


\section{Abstract}

Formation through new technologies represents the present and the future of every educational system. Taking into account the formational possibilities they offer, it is necessary to count with new formation areas for teachers, so that they can develop their professional expertise in a balanced manner with the highest chances for success. In this article an experience is presented in which both the actual professors as well as the future teachers have the possibility to complete their university formation through the "Oratory Classroom” of the University of Cantabria, in order to become great communicators.

\section{Key words}

Oratory - Virtual Education - Teachers' Formation - Oral Communication

\section{Las Nuevas Tecnologías de la Información y la Comunicación al servicio de la Educación.}

El uso de las Nuevas Tecnologías de la Información y la Comunicación (TICs) es un pilar importante de los métodos de enseñanza en cualquier nivel educativo y sus ventajas están siendo comprobadas cada día, por lo que se hace incuestionable su incorporación a los métodos de enseñanza actuales. Sin embargo el ritmo vertiginoso con el que avanza la tecnología requiere que los modelos de enseñanza evolucionen de igual manera, por lo que se pueden detectar algunos inconvenientes en su implementación.

El sistema educativo español, a través de la Ley Orgánica de Calidad de la Educación (LOCE, 2002) planteaba, en la exposición de motivos, y a través de los Reales Decretos que la desarrollan, la necesidad de incorporar las nuevas tecnologías dentro del curriculum básico, además de destacar la importancia de la comunicación tanto oral como escrita:

“La plena integración de España en el contexto europeo comporta una mayor apertura y exige un mayor grado de homologación y flexibilidad del sistema 
europeo. Exige también que los alumnos puedan adquirir destrezas que, como la capacidad de comunicarse -también en otras lenguas-, la de trabajar en equipo, la de identificar y resolver problemas, o la de aprovechar las nuevas tecnologías para todo ello, resultan hoy irrenunciables”.

“ En el curriculum de educación primaria destaca el impulso que se da a la utilización de las tecnologías de la información y la comunicación, el dominio de la expresión oral y al tratamiento de la lectura y de la comprensión lectora, siempre desde el marco que determinan las enseñanzas comunes establecidas. La necesidad de la utilización adecuada por los alumnos de las tecnologías de la información y la comunicación como herramienta de trabajo, la capacidad de expresarse correctamente y la conveniencia de una comprensión autónoma de los textos justifican suficientemente el modo en que se han incluido estos aspectos en el curriculum de educación primaria”.

Posteriormente, la Ley Orgánica de Educación (LOE, 2006) hace hincapié en el fomento de experiencias de iniciación temprana en las tecnologías de la información y la comunicación, ya desde el segundo ciclo de la etapa de Educación Infantil (3-6 años).

La incorporación de las TICs en las aulas es un hecho en España, pero se ha de tener en cuenta que su completa implementación requiere la inversión de recursos en los centros escolares, pero no sólo recursos económicos, si no también recursos personales, debido a la necesidad de contar con docentes competentes en el manejo y la enseñanza del uso de estas tecnologías.

Los planes de formación de docentes que se desarrollan actualmente en las universidades españolas contemplan la asignatura de “Nuevas tecnologías aplicadas a la Educación” que se desarrolla durante un cuatrimestre (4,5 créditos), a través de la cuál los futuros docentes toman contacto por primera vez, en muchos casos, con las TICs. Parece evidente que esta formación no es suficiente, puesto que si realmente se pretende que los profesores faciliten la utilización de las TICs a los alumnos para que las utilicen como herramienta de trabajo, será preciso que su formación sea más completa en este ámbito. En este sentido señala Salinas (2000) que es necesario un cambio de actitud general hacia las TICs y hacia su importancia tanto en el sistema educativo, como en la sociedad en general. Por lo tanto, las TICs pueden concebirse como un recurso y, a su vez, una estrategia para conseguir un aprendizaje diferente, más rápido y eficaz. 
Por otra parte, cabe señalar que el ritmo vertiginoso con que las TICs avanzan requieren de una formación continua de todo aquel que las maneja o pretende hacerlo, por lo que es preciso el reciclaje continuo del profesorado, no sólo para la utilización de los diferentes programas educativos que puede aplicar en el aula, sino porque es necesario un cambio en la forma de enseñanza, en su método, en su forma de entender la enseñanza.

Este cambio supone un reto para el profesor, tal y como señala Durán (2005), y supone también lo que podría entenderse como una “segunda alfabetización” imprescindible para la vida cultural y social. Las TICs están posibilitando la aparición de nuevos entornos de enseñanza aprendizaje por lo que las instituciones educativas deben afrontar el desafío de los nuevos medios, a riesgo de verse relegadas.

\section{El Proceso de Comunicación.}

Sin duda, la característica más importante de un buen profesor es su capacidad comunicativa, sobre manera si tenemos en cuenta que para lograr mejorar la calidad de la educación, en cualquier nivel, es preciso que la comunicación que el docente establece con sus alumnos sea eficaz. Por lo tanto, los autores coinciden con Amayuela, Colunga y Alvárez (2005) cuando afirma que es preciso considerar que la comunicación es el vehículo esencial del proceso enseñanza aprendizaje.

En todo proceso de comunicación es fundamental tener en mente el correcto funcionamiento de tres aspectos: la voz, la personalidad y el lenguaje que utiliza quien comunica. Para profundizar en estos temas se puede recurrir a los trabajos publicados por Del Barrio y Borragán (1998, 1999, 2004, 2005). En ellos se analizan las diferentes condiciones que precisa cualquier comunicación y que se pueden sintetizar en:

\section{En cuanto a la voz y las palabras:}

1. Claridad. Las palabras tienen que ser claras. Hay que mover más la lengua para hablar claro, lo que requiere sentirlo (aprovechando, por ejemplo, el frío del hielo o de otro objeto dentro de la boca, utilizando un hilo...) y crear hueco. Y después hablar como si no se tuviese nada dentro de la boca. 
2. Volumen adecuado al contexto. Se debe conseguir mucha resonancia para que las palabras tengan más consistencia. Hablar en espacios pequeños, como un baño, hace sentir de forma más clara la resonancia.

3. Ritmo adecuado. La velocidad del habla normal es de 150 palabras por minuto. Una velocidad lenta aporta monotonía, una excesivamente rápida provoca cansancio, confusión y aturdimiento. Hay que adecuar la velocidad a la comprensión del que te escucha. ¿Cómo descubrirlo? Por la forma en que te mira. Lo mejor es cambiar las velocidades para dar más vida a lo que se dice.

4. Ayudarse del cuerpo. La expresión corporal, el gesto, crea las pausas cuando hablamos y transmite intenciones. Hablar es una acción de todo el cuerpo. La mayoría de las veces, incluso, dice más el cuerpo que las propias palabras. En realidad, los gestos mínimos, especialmente de la cara y los ojos, son los que más van a impactar en el que tenemos enfrente. Conocer el lenguaje gestual te permite expresar con más profundidad y con más seguridad.

5. Saber jugar con la voz. Hay que hablar manifestando nuestras intenciones, subiendo y bajando los tonos, jugando con la duración de las vocales y con las pausas, es decir, dar vida, dotar de intenciones al mensaje. Quien sabe mover el cuerpo hace que la voz juegue sola.

\section{En cuanto a la personalidad:}

1. Autoestima. Para comunicar es fundamental tener una valoración de uno mismo. La autoestima influirá en nuestra seguridad así como en el rendimiento de lo que nos propongamos.

2. Empatía. Es necesario atender y hacer ver que entendemos la situación del otro, sin tener que identificarnos necesariamente con ella, pero intentando "ponernos en su lugar”. Debe ser un ejercicio cotidiano y realizado sin ninguna violencia. Contacto visual y escucha activa son las herramientas para obtener un grado óptimo de empatía, creando constantemente un clima de confianza y tranquilidad alrededor.

3. Sintonía. Tan importante como el mensaje es el interés que tiene en ello el que lo escucha. ¡Solo se escucha con atención aquello que nos interesa! Hay que saber 
reaccionar ante las señales de atención o de distracción del público. El valor de la comunicación lo da el que escucha.

4. Autenticidad. Sólo cuando creemos en lo que estamos comunicando podemos transmitir convicción e influir en los demás. ¡Para convencer hay que vivir tu propio mensaje! No digas cualquier cosa, expresa lo que llevas dentro.

5. Autocontrol. Una comunicación eficaz y positiva implica, también, un adecuado nivel de ejecución. Para esto es conveniente mantener controlados los niveles de ansiedad, confiando en que comunicar es siempre un privilegio.

6. Asertividad, entendida como la propia capacidad de autoafirmación, de expresión directa de los propios sentimientos, opiniones, derechos, etc... Ser asertivo es sentirse mejor con uno mismo y demostrar que se está vivo.

\section{En cuanto al mensaje:}

1. Preparación. La clave del éxito se asienta en la preparación pausada. Una comunicación debe estar siempre muy "posada”. No es aconsejable montar el caballo de la improvisación, te tirará al suelo.

2. Corrección. Utilizar un lenguaje adecuado al público, claro y sencillo, pero siempre correcto. ¡Cultivar la lectura de la buena literatura!.

3. Orden. Mantener el esquema clásico de “Inicio, Desarrollo, Conclusión”: es lo que los demás esperan y además, lo más eficaz.

4. Claridad de las ideas. Marcar adecuadamente los tiempos. Cada momento del discurso tiene su propia dinámica y es preciso respetarla. Vibrar en cada momento y hacer así vibrar al que escucha.

5. Novedad. Ser creativo, original, sobre todo al principio, para ganar la atención, para motivar y cautivar. 


\subsection{La Comunicación en el Aula.}

Para analizar cómo se produce la comunicación en el aula es necesario tener en cuenta que el profesor posee una autoridad sobre el alumno, y un control del resultado final del proceso didáctico, que se materializa en la calificación final, y que sin duda condiciona todo el proceso comunicativo. En este sentido ya se oyen voces que apuntan hacia la necesidad de establecer una línea de autoridad más horizontal, de manera que se eliminen algunas de las barreras que dificultan la comunicación docente - alumnos, establecidas tradicionalmente.

Sin embargo, a pesar de esta diferencia de poder, definida como relación asimétrica por Camaraco (2005), lo interesante es que tanto el docente como el alumno tengan presente que el objetivo común que debe lograrse es el aprendizaje del alumno, obteniendo el máximo rendimiento. Es decir, el profesor no debe centrarse exclusivamente en transmitir, de la forma más eficaz posible, su materia, sino en que el alumno lo aprenda, y que ese aprendizaje tenga un carácter significativo.

Para lograrlo, el docente debe convertirse en un guía, un apoyo, del aprendizaje del alumno, llegando a ser un comunicador eficaz del conocimiento, de las actitudes y valores necesarios para que el alumno logre ser un ciudadano libre, responsable, democrático, íntegro...

Independientemente de la estrategia formativa que se utilice, el profesor ha de asegurar la comunicación en clase (Núñez, 2004), animando el verdadero proceso de comunicación. Para ello se deben cuidar especialmente tres aspectos:

- La voz: tan importante es modificar la voz para lograr comunicar al alumno de forma que le atraiga y le motive, como evitar un mal uso, que puede tener consecuencias perjudiciales en el futuro.

- Control visual: el contacto visual con los alumnos, con todos los alumnos, es fundamental para lograr una comunicación eficaz. El contacto visual debe establecerse a lo largo de todo el discurso, puesto que la buena orientación de la mirada permite la captación inicial de la atención de los alumnos, además de ayudar a mantenerla.

- Control corporal: el movimiento del cuerpo del profesor transmite tantos mensajes como la propia voz (comunicación no verbal), por lo tanto controlar el cuerpo, de manera que su expresividad sea congruente con el discurso, es fundamental para asegurar el éxito de la comunicación. 
Cuando un profesor es capaz de establecer con sus alumnos una comunicación verdaderamente educativa, según Camacho y Sáenz (2000) se cumplen los siguientes requisitos:

- La comunicación se basa en la confianza mutua y no en las relaciones de dominio sumisión. La confianza mutua se basa en el entendimiento y la mutua comprensión.

- La comunicación se produce tanto en situaciones formales (más habituales en el aula), como en situaciones informales (tanto dentro como fuera del aula).

- Es posible la libre expresión de las ideas y de las manifestaciones personales.

- Se favorecen frecuentes intercambios de los papeles de emisor y receptor, en los que la comunicación fluye en todas direcciones.

\section{El Aula de Oratoria de la Universidad de Cantabria}

En los apartados anteriores se han expuesto una serie de argumentos que pretenden resaltar las ventajas de la aplicación de las TICs al ámbito educativo, así como la necesidad de la formación de los docentes en su uso y aplicación para garantizar una correcta implementación en las aulas. Por otro lado, se ha tratado de señalar la importancia de la interacción entre profesor y alumnos en el aula como clave del proceso educativo, en la cuál la comunicación juega un papel fundamental; así como la conveniencia de que los docentes reciban una formación inicial y permanente adecuada, puesto que el conocimiento y la práctica de las técnicas de la comunicación eficaz incrementan el impacto de la actividad docente y, por tanto, contribuyen a un aprendizaje más firme.

Ante este panorama, resulta conveniente presentar la aportación del grupo de investigación “Isla de Mouro” de la Universidad de Cantabria y otros colaboradores, tras la creación del “Aula de Oratoria” (www.auladeoratoria.com), el pasado mes de febrero de este mismo año, como una oportunidad para complementar la formación de los docentes, a través de las TICs, así como de los alumnos y de profesionales de la voz.

El Aula de Oratoria nace, bajo la dirección conjunta del profesor de la U.C. Dr. José A. del Barrio y del Dr. Alfonso Borragán (médico foniatra), como fruto de un convenio entre la Universidad de Cantabria (Fundación Leonardo Torres Quevedo) y la entidad bancaria La Caixa, cuyo objetivo es fomentar el arte de hablar entre docentes y alumnos de la Universidad de Cantabria, de otras Universidades y del personal de la Caixa, buscando extender también su actividad a otros 
profesionales que tengan que utilizar la comunicación y el lenguaje para sus actividades laborales, docentes, sociales o científicas y que demanden este servicio.

A través del Aula de Oratoria cualquier persona puede aprender a comunicarse de forma eficaz, para lo cuál se ofrece la posibilidad de indagar sobre la situación de cada uno en algunos aspectos capitales del acto comunicativo, a través de unos cuestionarios on-line. A partir de los resultados obtenidos en los cuestionarios, cada usuario tiene la posibilidad de mejorar los aspectos comunicativos necesarios en los cursos de formación que se ofrecen. Actualmente los cursos se imparten de forma presencial, si bien, próximamente se dará el salto a los cursos virtuales, facilitando el acceso a un mayor número de personas, en los que se profundizará en las técnicas comunicativas.

Otra de las aportaciones originales del Aula de Oratoria es ofrecer una serie de conferencias a través de su página web, tanto de oradores famosos, como de otros conferenciantes de la propia universidad, las cuáles estarán en breve disponibles. Además de poder visualizar y escuchar las conferencias, podrán ser analizadas a partir de unos indicadores, de manera que puedan servir de referencia para toda aquella persona que quiera aprender o mejorar el arte de comunicar.

\subsection{Objetivos}

El nuevo Aula de la Universidad de Cantabria se plantea como propósito hacer de los alumnos, de los profesores y de otros colectivos que así lo demanden, grandes comunicadores, utilizando técnicas que adquieran en el Aula de Oratoria.

Entre los objetivos específicos se plantean los siguientes:

1. Aprender a transmitir de una forma más eficaz los conocimientos específicos.

2. Mejorar la expresión verbal para ser más competitivos profesionalmente.

3. Realizar perfiles individuales de la capacidad de comunicación.

4. Reforzar los puntos fuertes de la comunicación.

5. Normalizar los puntos débiles a través del aprendizaje de estrategias.

6. Potenciar la voz de cada uno y enriquecer su competencia lingüística.

7. Crear un foro de conferencias permanente en la Universidad de Cantabria.

8. Extender estas actividades a colectivos profesionales interesados. 
Aprender el arte de la comunicación es complejo: se requiere la ayuda de expertos y tiempo. Hacer cursos aislados sobre voz, control de ansiedad, lenguaje... no suele ser eficaz. Los buenos resultados en este campo requieren un marco teórico (tener conceptos claros) y mucha práctica:

- Realizar muchas horas de auto-observación mediante sistemas digitales de grabación en alta fidelidad.

- Poner en práctica todo lo que se aprende a través de conferencias con público.

- Disponer de buenos modelos para el aprendizaje por ósmosis.

- Corregir previamente todos los problemas ORL, foniátricos o logopédicos que cada uno tenga.

- Poseer una voz de gran calidad tímbrica y resistente a la fatiga vocal.

Si se mantienen estos principios básicos durante el tiempo adecuado, el resultado será la formación de excelentes oradores.

\subsection{Aplicaciones al ámbito educativo.}

El Aula de Oratoria tiene su máxima actuación en el ámbito educativo, puesto que, como ya se ha señalado, mejorar las competencias comunicativas de docentes y de alumnos son sus principales objetivos.

\subsubsection{Mejorar la comunicación de los docentes}

Mejorar, optimizar y potenciar la comunicación de un docente puede resultar paradójico a simple vista. Sin embargo, un docente que no sea un gran comunicador tendrá más difícil la adecuada transmisión de sus conocimientos e intuiciones investigadoras. Esta habilidad, o incluso arte, no es un don natural.

Un docente dotado de una buena capacidad comunicativa será capaz de:

- Lograr la atención del alumno en el aula.

- Transmitir por ósmosis la capacidad de expresión y empatía: dotar al alumnos de forma espontánea de una gran capacidad comunicativa. 
- Poseer una potentísima herramienta para atraer y motivar.

- Reforzar, con el éxito, el sentido y la ilusión por lo que hace.

La voz es la herramienta para materializar la comunicación oral. Si la herramienta no está afinada, el mal funcionamiento puede suponer que cualquier acto de habla signifique dolor, cansancio, sobreesfuerzo. La fatiga vocal genera menor calidad de comunicación, menor calidad en la docencia, mayor desgaste personal y desmotivación ( un tercio de las bajas producidas entre los docentes se deben a problemas de voz). Todo esto conduce inexorablemente hacia la depresión.

\subsubsection{Mejorar la comunicación de los alumnos}

Formar alumnos implica formar personas, ciudadanos, profesionales, con una serie de valores, competencias y de conocimientos que tendrán que ir demostrando a lo largo de su existencia. Si a estas personas se les forma para que sean buenos comunicadores, esto repercutirá positivamente en su desarrollo personal, en la adquisición de técnicas eficaces y cooperativas para el trabajo en grupo o en la creación de situaciones positivas en las relaciones personales, etc. Además de todas estas ventajas, no se ha de olvidar que también obtendrá beneficios en su futuro desempeño profesional, tales como:

- Todas las empresas aprecian enormemente al empleado o directivo que sabe comunicar lo que conoce, vive y siente.

- Abre puertas tanto al individuo como a la empresa que representa.

- Lo que hace, investiga, promueve o fabrica convence o no según como se represente.

- Una adecuada comunicación sólo aporta beneficios en el amplio espectro laboral.

\section{3. ¿Cómo ser un Orador Eficaz?}

La Oratoria puede definirse como un arte en el que hay que "hacerse creer”, esto es:

- Hacer vivir al que escucha lo que el comunicador siente y piensa, sus intenciones.

- Mostrarse como uno es.

- Mantener viva la atención y el interés por lo comunicado

- Transmitir con todo el cuerpo: la voz, la expresión, la postura. 
Todo ello obliga al orador eficaz a cumplir una serie de requisitos:

- Tener claro lo que se quiere transmitir.

- Conocerse, aceptarse y saber sacar lo mejor de uno mismo.

- Adivinar lo que el otro siente, quiere y piensa.

- Poseer no un lenguaje sino muchos.

- Saber jugar sobre un escenario con los otros.

Un orador eficaz posee una serie de características que se pueden sintetizar en tres: es consciente del uso adecuado que ha de ejercer con su voz, posee los conocimientos suficientes sobre los que va a comunicar y por lo tanto estructura perfectamente los mensajes que emite, y su personalidad es equilibrada, lo que le permite conocer a los demás y adaptarse a las diferentes situaciones.

Desde el Aula de Oratoria se ofrece la posibilidad no sólo de mejorar las técnicas comunicativas, sino de conocer cuál es la capacidad comunicativa de cada persona. Gracias a las aplicaciones de las TICs, se han diseñado una serie de cuestionarios a las que cualquier persona puede acceder a través de la página web, se ofrece la posibilidad de realizar cursos virtuales a los que pueda tener acceso un mayor número de personas, así como un “Analizador Gráfico del Habla” (AGHA), disponible a través de la página web, de manera que cualquier persona interesada en analizar y mejorar su capacidad comunicativa pueda conocer de una forma más precisa si su comunicación es eficaz o necesita mejorar, analizando aspectos de la propia voz, como la onda melódica, la frecuencia, el juego vocal y la velocidad de las palabras.

\subsubsection{Cuestionarios on-line.}

En el acto comunicativo existen algunos aspectos fundamentales en los que conviene conocer la situación de cada persona, en cuanto a la capacidad comunicativa, el impacto que causa en el que le escucha, el miedo escénico que siente ante el hecho de hablar en público, si su voz corre algún riesgo o si la utiliza de forma correcta, así como el nivel de estrés con el que vive. Para ello se han diseñado una serie de cuestionarios a través de los cuáles las personas interesadas en mejorar su capacidad comunicativa pueden comprobar la situación en cada momento, así como comprobar cuáles son los aspectos que conviene mejorar o potenciar. Son los siguientes: 
- Estudia tu Comunicación. Conoce los pilares sobre los que se basa la comunicación.

Para mejorar la práctica comunicativa es necesario conocer la calidad del mensaje que se emite, la utilización de la voz y de las palabras durante el discurso, así como la propia personalidad y el estilo personal en el acto comunicativo.

- Valora el impacto de tu comunicación. Examina el impacto de tu comunicación.

Se pretende valorar una serie de parámetros básicos que todo buen comunicador ha de tener presente: la Atención, la Motivación, el Interés, la Estructura, el Efecto de la Comunicación y la Actitud.

- Descubre si sientes Confianza como Comunicador. Considera cómo incide el público en ti.

Se pretende que cada persona descubra si siente confianza en sí mismo cuando habla ante un público numeroso, analizando sus sensaciones antes de una exposición oral, durante la exposición y después de la exposición.

- Conoce tu Riesgo Vocal. Valora el riesgo que corre tu voz.

Existen muchos elementos que pueden alterar el buen funcionamiento de la voz. Algunos son conocidos, como el mal uso, y otros desconocidos (como los causados por problemas digestivos o por chupar caramelos de menta). Existen, además, creencias infundadas, que van pasando de unos a otros, sobre qué cosas influyen en la voz: por ejemplo, las bebidas frías. El frío no debería afectar a la voz y, cuando lo hace, la causa se encuentra más bien en la ingestión de la bebida fría en un sitio de ruido (siendo en realidad el ruido el factor desestabilizador) o en la existencia de un problema local laríngeo que lo explica (pólipo laríngeo, lesiones congénitas...).

En la producción de la voz intervienen muchos sistemas y en cada uno de ellos, a su vez, inciden múltiples variables: cuando la voz se estropea no suele deberse a una única malfunción sino a varias. El trastorno vocal surge cuando se sobrepasa cierto umbral crítico en el factor (o la suma de factores) de riesgo. Conocerlos, por tanto, resulta de gran importancia de cara al buen uso y a la prevención. 
Los factores de riesgo se pueden agrupar en 6 bloques:

1. Bloque del mal uso vocal, que genera traumatismos y lesiones directamente en el órgano vocal.

2. Bloque de los conflictos emocionales y afectivos, causa de una rigidez del sistema y, por consiguiente, fuente de problemas de voz.

3. Bloque de factores diversos. De modo general, cualquier cambio en el tracto vocal conlleva modificaciones en la voz.

4. Bloque de los cambios en el organismo, que facilitará la malfunción del tracto vocal.

5. Bloque de los cambios en la lubrificación. Las cuerdas vocales se mueven con tanta rapidez que una lubrificación escasa o inexistente hace inevitable las lesiones de quemadura por roce.

6. Bloque de los factores constitucionales, por malformación del tracto vocal, causa muy frecuente

- ¿ ¿Con cuánto estrés vives? Descubre el nivel de estrés con el que vives.

Vivir con altos niveles de estrés condiciona cualquier acto de un ser humano. La comunicación no se libra de ello: las ideas no fluyen, los mensajes no se adaptan con precisión al que escucha..., por lo que se recomienda reducir y controlar el estrés para comunicar de forma eficaz. En este cuestionario se estructura el estrés en indicadores físicos, indicadores de los procesos mentales, indicadores emocionales e indicadores conductuales.

\subsubsection{Cursos formativos presenciales.}

A lo largo del año 2006 se están ofreciendo diversos cursos a través del Aula de Oratoria, de forma presencial. Algunos de los cursos presenciales son los siguientes:

- $\quad$ Atraer Hablando: Técnicas para hablar en público. 
Dirigido a alumnos de los últimos cursos de la Facultad de Derecho de la Universidad de Cantabria. Se trata de un curso de iniciación para personas no habituadas a hablar en público.

- $\quad$ Atraer Hablando: Comunicación Eficaz en el Aula

Dirigido a profesores de la Universidad de Cantabria.

- Atraer hablando: el impacto de la comunicación.

Dirigido a quienes hablan en público, pero quieren ganar en impacto.

- Atraer hablando y escuchando.

Dirigido a personas que atienden al público.

\subsubsection{Cursos formativos virtuales.}

El Aula de Oratoria de la Universidad de Cantabria se caracteriza por la aplicación de las TICs al proceso de formación de aquellas personas interesadas en mejorar su capacidad comunicativa. Los cursos virtuales serán una de las señas de identidad del grupo de profesionales que trabajan en el Aula y se irán ofertando de forma continuada. Con este propósito se ha diseñado ya el curso “El Arte de Hablar. Atraer Hablando” que estará en breve disponible.

Se trata de un curso virtual de oratoria y comunicación eficaz para mejorar la capacidad de comunicación de cualquier docente. Está diseñado para la autoformación y para el aprendizaje a distancia mediante tutorización personalizada de cada alumno. Los destinatarios son profesores de cualquiera de las áreas educativas.

Los materiales necesarios para seguir el curso son un CD-ROM que se facilitará a los alumnos, y que se compone de una guía del alumno y de un documento multimedia con los contenidos del curso. Se recomienda utilizar un equipo informático compuesto por un ordenador Pentinum III o superior que cuente con lector de CD, tarjeta de sonido y altavoces y tarjeta de vídeo SVGA.

\section{Conclusiones}

- Las Nuevas Tecnologías de la Información y la Comunicación poseen actualmente un papel relevante en los sistemas de enseñanza, con una aplicación en el curriculum escolar que reporta innumerables ventajas tanto a alumnos como a profesores. Si bien es cierto que el 
nivel de conocimiento y uso de las TICs por parte de los docentes requiere de una formación inicial y continua en constante actualización.

- La comunicación es uno de los elementos más importantes para asegurar el éxito del proceso enseñanza - aprendizaje, por lo que se debe tener en cuenta el correcto funcionamiento de los múltiples factores que lo determinan, como la voz, el estilo personal, el lenguaje verbal y no verbal, la capacidad de escucha, la motivación... La comunicación eficaz es un arte, con el que no se nace, sino que se aprende, se mejora, por lo que requiere de un proceso de formación.

- El Aula de Oratoria de la Universidad de Cantabria supone una oportunidad para ayudar tanto a alumnos, como a docentes, como a otros colectivos interesados, para lograr que las personas tengan una adecuada capacidad comunicativa, utilizando para ello las TICs, como herramienta que facilita el acceso a toda persona interesada en mejorar su oratoria, a través de los cuestinarios on-line, los cursos virtuales o el AGHA.

\section{Bibliografía}

- AMAYUELA, G., COLUNGA, S. Y ALVAREZ, N., 2005, “Docencia universitaria y comunicación educativa”. En Contextos educativos. Revista digital de educación y nuevas tecnologías, ${ }^{\circ}$ 36, ano VI www.contexto-educativo.com-ar/2005/3/nota-o6 consultado el $30 / 05 / 2006$

- BARRIO, J.A. y BORRAGÁN, A., 2004, Trastornos de la comunicación. Una aproximación interpersonal. Volumen 1. Santander: TGD.

- BARRIO, J.A. y otros., 2005. Nuevos contextos psicológicos y sociales en educación. Buscando respuestas. Badajoz. Psicoex.

- BORRAGÁN, A., BARRIO, J.A., y GUTIÉRREZ, J.N., 1998, El juego vocal. Para prevenir problemas de voz en contextos escolares. Santander: Gobierno de Cantabria.

- BORRAGÁN, A., BARRIO, J.A., y GUTIÉRREZ, J.N., 1999, El juego vocal. Para prevenir problemas de voz. Málaga. Aljibe. 
- BORRAGÁN, A. y otros., 2005, “Jugar con la voz para prevenir problemas y atraer la atención de los alumnos” en VICENTE, F. y otros. Psicología y Educación: Nuevas investigaciones. Badajoz: Psicoex, pp. 13-28.

- CAMACHO, S. y PEREZ, O. 2000. Técnicas de comunicación eficaz para profesores y formadores. Alcoy. Ed. Marfil.

- CAMARACO, Z., 2005, “El aula de clase: contexto para la elaboración de la imagen”. En Educere, $n^{\circ}$ 30, pp. 311-316.

- DURAN, A.S., 2005, "El uso de las nuevas tecnologías en los centros educativos: realidad o ficción”, en Congreso Internacional Virtual y Presencial sobre el Profesorado ante el Reto de las Nuevas Tecnologías en la Sociedad del Conocimiento. Universidad de Granada.

- LEY ORGÁNICA 10/2002, de 23 de diciembre, de CALIDAD DE LA EDUCACIÓN. ( BOE de 24 de diciembre de 2002).

- LEY ORGÁNICA 2/2006, de 3 de mayo, de EDUCACIÓN (BOE de 4 de mayo de 2006)

- NÚÑEZ, T., 2004, “Asegure la comunicación en clase y negocie acuerdos de aprendizaje”, en VILLAR, L.M. Programa para la mejora de la docencia universitaia. Madrid: Pearson - Prentice Hall.

- REAL DECRETO 115/2004, de 23 de enero, por el que se establece el curriculum de Educación Primaria. (BOE de 7 de febrero de 2004).

- SALINAS, J., 2000: “El rol del profesorado en el mundo digital”, en DEL CARMEN, L., Symposium sobre la formación inicial de los profesionales de la educación. Universidad de Gerona.

- www.auladeoratoria.com 